\title{
TRACZ - Testing Robotic Applications for Catching in Zero-g as the first step to research jamming phenomenon in the non-Earth conditions
}

\author{
A. Graja, A. Bojda, A. Gorgolewski, K. Bęś, K. Mirek, Ł. Chojnacki, and A. Sil \\ Wroclaw University of Science and Technology, \\ Wrocław, Poland, \\ e-mail: info@tracz-rexus.pl
}

\begin{abstract}
TRACZ - Testing Robotic Applications for Catching in Zero-g is a fully autonomous mechatronic experiment, launched on March 19, 2019, on board the REXUS rocket, during the 26th Campaign of the REXUS/BEXUS Programme. The main goal of the experiment was to investigate the jamming phenomenon in the space environment (microgravity and vacuum) as well as to construct a version of industry device known as jamming gripper, capable of operating in conditions where negative pressure (physical basis of operation in Earth-like conditions) cannot be obtained due to the presence of only a residual atmosphere. The structure was designed as 1-DOF manipulator, whose effector is an elastic membrane that stimulates jamming of ground coffee particles inside. During the suborbital flight, a series of catches were performed on a single object and the force with which the object is held was measured. The results were compared with an on-ground experiment.
\end{abstract}

Keywords: jamming phenomenon, gripper, rocket experiment, rocket payload, catching space objects, REXUS/BEXUS Programme

\section{THE JAMMING PHENOMENON}

A jamming phenomenon, described as transition of grainy substances to solid-state under specific conditions is still a relatively unexplored issue. Theorems that classify the jamming phenomenon as a new example of a phase transition generate a need for a better description, modelling and definition of conditions of its occurrence. The jammed solid can be fluidized under the influence of temperature, vibration or mechanical stress. The generality of the jamming transition led to the proposal of a unifying description, based on a jamming phase diagram [1] (Fig. 1).

\section{Motivation}

Grasping different objects by robots in space conditions is in many cases neither effective nor convenient. Lack of general-purpose device which can grab differently shaped and sized elements made from various materials is one of many issues in space missions. A classical approach towards gripping objects by human-like rigid effector requires sophisticated trajectory planning algorithms, numerous sensors, and complicated mechanical design. Another approach is to use soft, elastic materials manipulated by pressure to adjust to an irregular-shaped object and catch it. Soft grippers are less complicated in construction and use, furthermore, they seem to be more all-embracing.

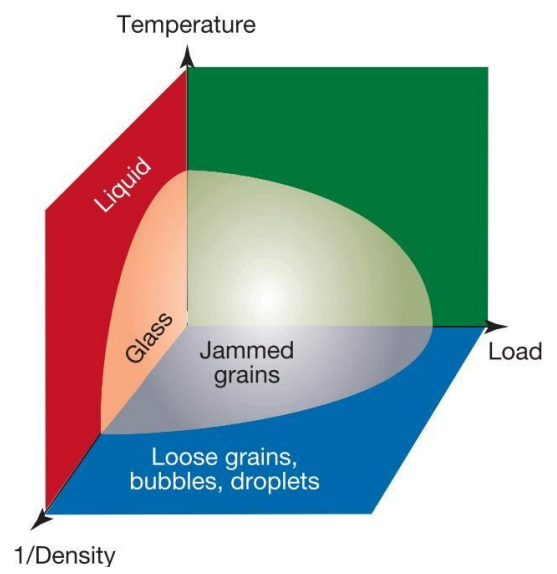

Fig. 1. Jamming phase diagram [1]

III. GRIPPER STRUCTURE

The inspiration to create an unconventional tool came from new trends in the manufacturing industry. The Empire Robotics company has commercialized jamming-based robotic gripping technology in a product called VERSABAL[2]. This type of gripper is based on the jamming transition in granular systems. Pressurized membrane (e.g. neoprene in latex form) filled with granular medium (e.g. ground coffee) is adjusting to object, then depressurization of the membrane is performed 
and granular medium (and therefore also the object) gets jammed - the object can be manipulated (Fig.2).

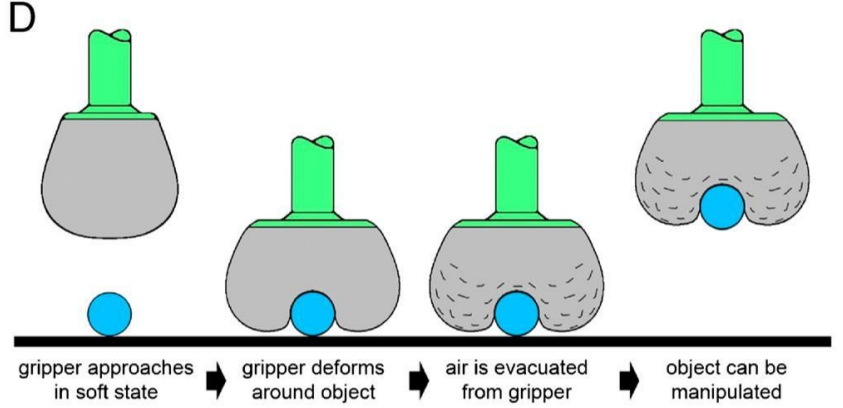

Fig. 2. Universal gripper based on the jamming of granular material [3]

There is no prior documented case of testing such technology in space conditions (microgravity and vacuum). Industrial jamming grippers might work with negative (down to $-0,85$ bar) and positive (up to 6,2 bar) pressures [4] to increase gripping forces. It is not possible to mimic this approach in a vacuum. It was also determined that behaviour of granulate in zero-g is unknown and may have a significant impact on the operation of the gripper. Moreover, in zero-g, minimal grasping forces needed to effectively manipulate the objects are significantly lower. Therefore suborbital flight under the REXUS/BEXUS programme was chosen as a test method as it offers exposure to both microgravity and vacuum for substantial amount of time, allowing to perform several, statistically significant, gripping procedures.
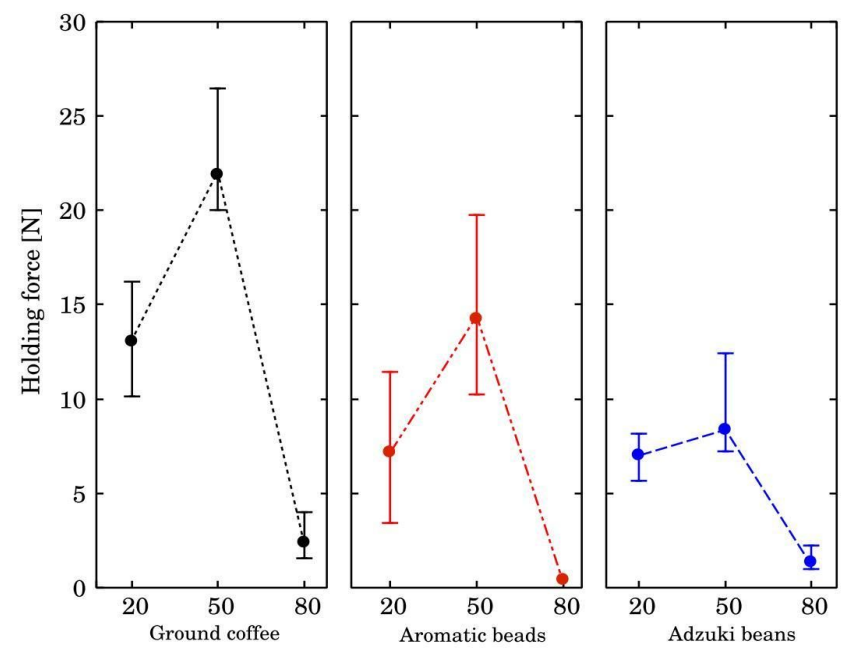

Filling ratio of material [\%]

Fig. 3. Relationship between filling ratio and holding force of the gripper [5]

As commercial off-the-shelf jamming grippers are not adapted to space conditions, it was decided to design and build the effector from scratch. Based on the previous research, ground coffee was selected as the granulate (Fig. 3) [5]. For the membrane material two-component silicone was selected and its shape was formed in the casting process using various forms. Initial idea assumed oval gripper shape (offering greatest versatility), however, after many tests on Earth, it was concluded that oval membrane, lacking negative pressure, could not generate sufficient jamming to prevent the membrane from returning to its equilibrium position (oval shape), pushing out the object. Due to these issues, it was decided to change the shape of the membrane to the one shown in the photo of one of the prototypes (Fig.4).

Pressure control system consisted of two pressure regulators and two normally closed solenoid valves. Compressed air (reduced to around $60 \mathrm{kPa}$ with pressure regulators) was supplied from the tank to make grains loose in inflation phase. For air evacuation phase, outlet valve was opened to remove air from the membrane due to a natural pressure equalization (as the differential pressure is basis of operation, this method works in both a vacuum and an Earth's atmosphere). Membrane and pneumatic system were tightly connected with fastening and sealing elements printed from epoxy resin with SLA printing technology.

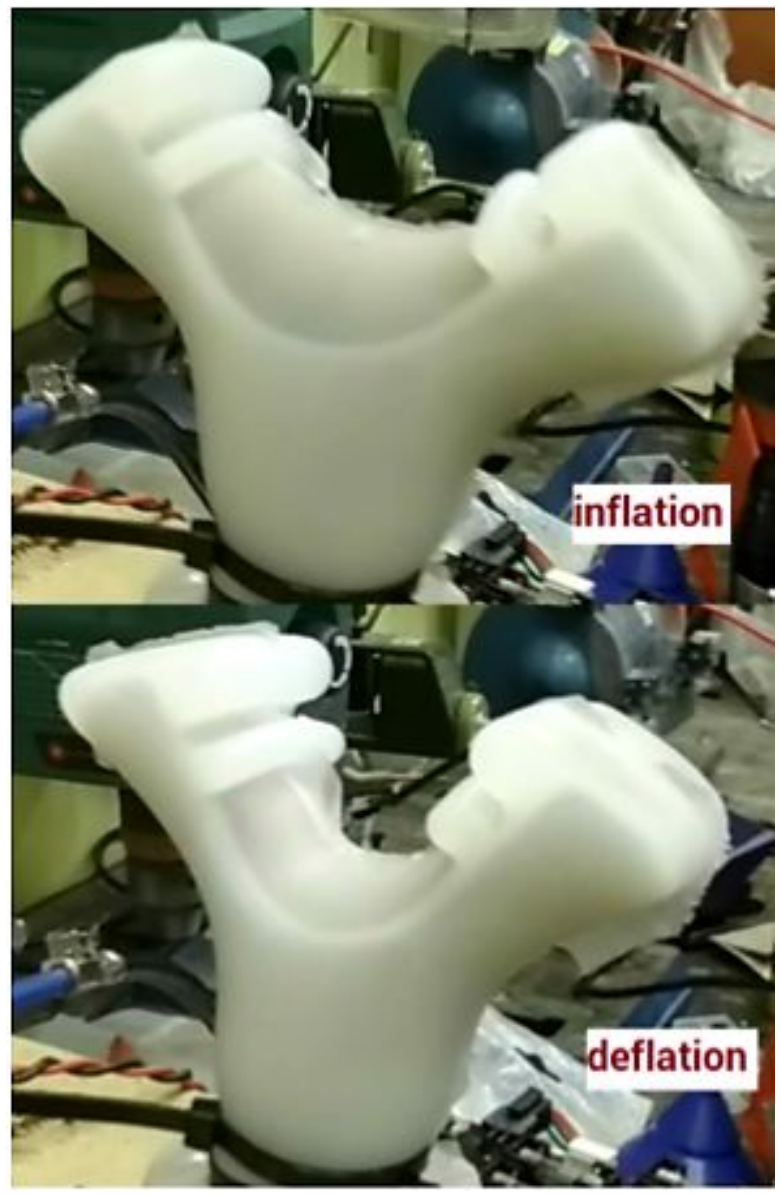

Fig. 4. End effector in two states (prototype)

\section{DATA ANALYSIS PLAN}

The data gathered during the experiment was used to evaluate the utility of using the jamming gripper to grasp objects in the outer space. This utility was defined in the 
following manner. Firstly, the gripper should be able to catch the object. It is determined by a success rate in percentage over all the possible trials. Secondly The pushing force $F p$ should be as low as possible to not push back the object being grabbed and from the other hand, the holding force Fh (Fig. 5) should be enough to move the object.

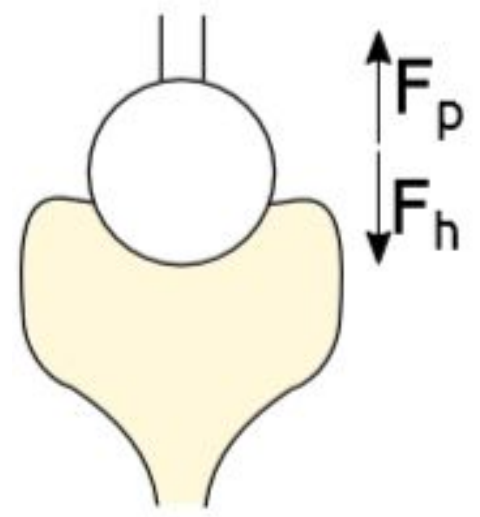

Fig. 5. A sketch of experiment setup

The experiment setup consisted of a set of sensors that allowed measuring all necessary parameters as the pushing force $F p$, the holding force $F h$ and environmental conditions. The most important was the piezoelectric load cell to which the gripped sample was attached. This sensor allowed to measure the pushing and holding force. The second one, a differential pressure sensor measured the overpressure inside the membrane.

\section{RESULTS AND DISCUSSION}

The main purpose of the experiment was to perform several grips with different pushing force $F p$ and observe the holding force $F h$. It was decided to approach the sample with two different pushing forces of $40 \mathrm{~N}$ and $50 \mathrm{~N}$. The results from the Earth experiment are shown in figure 6. As presented in table 1 , the average holding force in case of $40 \mathrm{~N}$ pushing force is $11.55 \mathrm{~N}$ with standard deviation of $0.29 \mathrm{~N}$, and in case of $50 \mathrm{~N}$ pushing force, it is $12.33 \mathrm{~N}$ with standard deviation of $0.17 \mathrm{~N}$. The effector achieved $100 \%$ success rate on Earth.

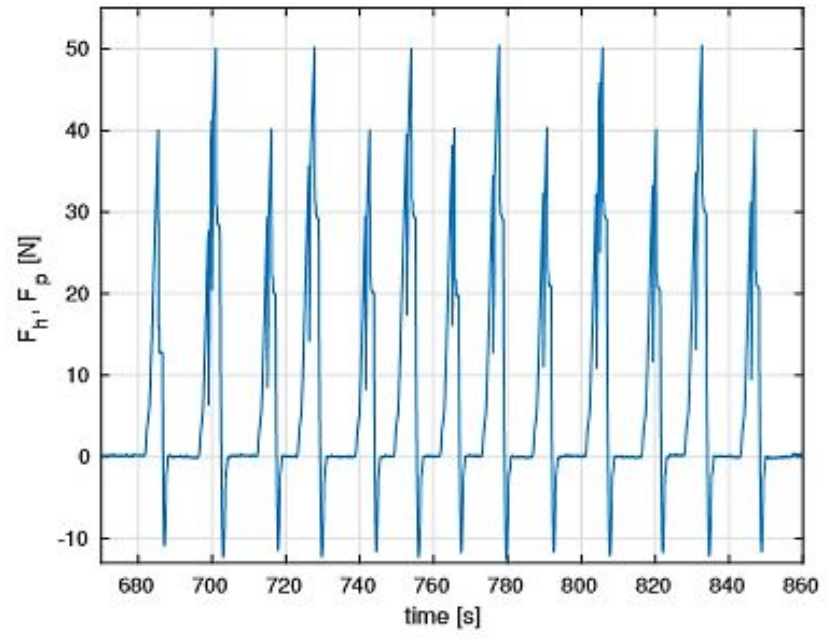

Fig. 6. Holding force $F$ h and pushing force $F p$ in time during Earth experiment

TABLE 1 HOLDING FORCES Fh FOR GIVEN PUSH FORCE Fp DURING EARTH EXPERIMENT

\begin{tabular}{|c|c|c|c|c|c|c|c|}
\hline $\mathrm{F}_{p}[\mathrm{~N}]$ & $\mathrm{F}_{h 1}[\mathrm{~N}]$ & $\mathrm{F}_{h 2}[\mathrm{~N}]$ & $\mathrm{F}_{h 3}[\mathrm{~N}]$ & $\mathrm{F}_{h 4}[\mathrm{~N}]$ & $\mathrm{F}_{h 5}[\mathrm{~N}]$ & $\mathrm{F}_{h 6}[\mathrm{~N}]$ & $\mathrm{F}_{h 7}[\mathrm{~N}]$ \\
\hline 40 & 10.94 & 11.48 & 11.68 & 11.77 & 11.70 & 11.73 & 11.59 \\
\hline 50 & 12.15 & 12.27 & 12.31 & 12.51 & 12.06 & 12.07 & - \\
\hline \\
$\qquad$\begin{tabular}{|c|c|c|c|}
\hline $\mathrm{F}_{p}[\mathrm{~N}]$ & Mean $[\mathrm{N}]$ & Std $[\mathrm{N}]$ \\
\hline 40 & 11.55 & 0.29 \\
\hline 50 & 12.33 & 0.17 \\
\hline
\end{tabular}
\end{tabular}

The results from rocket experiment are shown in figure 7. As presented in table 2 the average holding force in case of $40 \mathrm{~N}$ pushing force is $10.58 \mathrm{~N}$ with standard deviation of $0.19 \mathrm{~N}$, and in case of $50 \mathrm{~N}$ pushing force is $11.55 \mathrm{~N}$ with standard deviation of $0.23 \mathrm{~N}$. Also during the rocket experiment, the effector achieved $100 \%$ success rate.

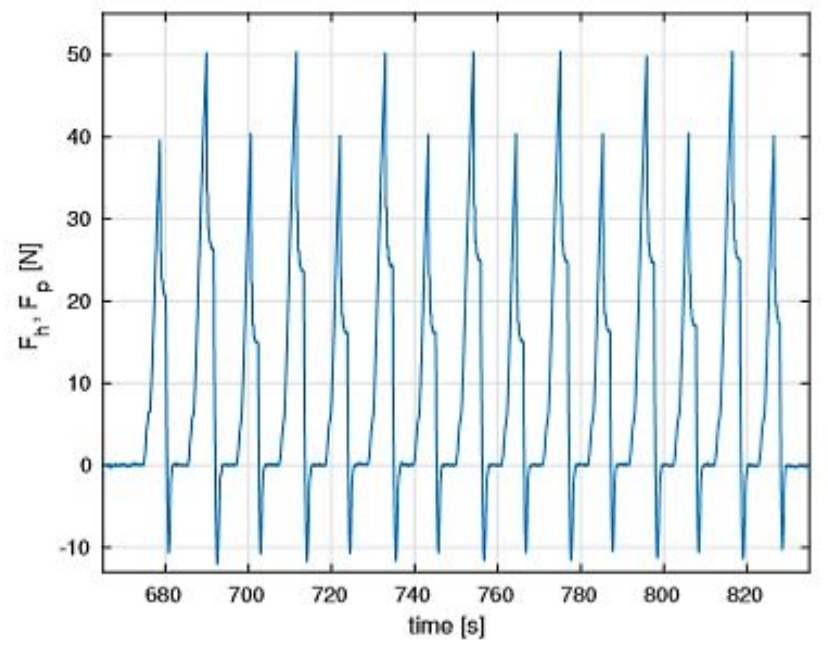

Fig. 7. Holding force Fh and pushing force Fp in time during Space experiment 


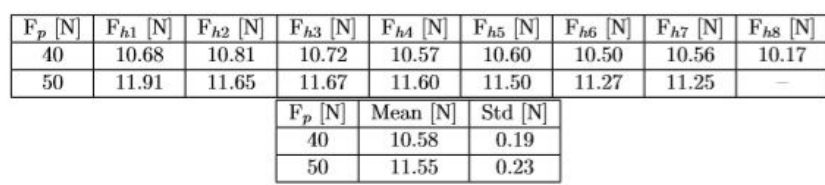

To generate around $10 \mathrm{~N}$ of holding force, the gripper has to push the sample with the force around $50 \mathrm{~N}$. The high pushing force can cause the object to be pushed away to the outer space. However, the series of experiments on Earth and in space show that the proposed gripper achieves similar performance in both environments, regardless of atmospheric pressure and gravity. However more experiments with different samples are needed to confirm it. Other effector shapes can also be explored, however, authors believe that another interesting (and perhaps more promising) idea is to extend soft gripper capabilities with use of smart materials, e.g. ferromagnetic material instead of jamming material to achieve the same gripping mechanism and to eliminate the aforementioned disadvantages of the jamming gripper.

\section{REFERENCES}

[1] V. Trappe*2, V. Prasad*, Luca Cipelletti*2, P. N. Segre*2 \& D. A. Weitz*, "Jamming phase diagram for attractive particles", Department of Physics and DEAS, Harvard University, Cambridge, Massachusetts 02138, USA, NATURE, VOL 411, 14 JUNE 2001.

[2] Amend, John; Cheng, Nadia; Fakhouri, Sami; Culley, Bill, "Soft Robotics Commercialization: Jamming Grippers from Research to Product". w: Soft robotics 3 (4), s. 213-222, 2016.

[3] Eric Brown, Nicholas Rodenberg, John Amend, Annan Mozeika, Erik Steltz, Mitchell R Zakin, Hod Lipson, and Heinrich M Jaeger. "Universal robotic gripper based on the jamming of granular material". Proceedings of the National Academy of Sciences, 107(44):18809-18814, 2010.

[4] David Charles Blackley. Polymer Latices: Science and Technology Volume 3: Applications of Latices. Springer Science \& Business Media, 2012.

[5] Takeshi Nishida, Daichi Shigehisa, Naoaki Kawashima, and Kenjiro Tadakuma. Development of universal jamming gripper with a force feedback mechanism. In Soft Computing and Intelligent Systems (SCIS), 2014 Joint 7th International Conference on and Advanced Intelligent Systems (ISIS), 15th International Symposium on, pages 242-246. IEEE, 2014. 